\title{
Comparison of Image Processing Techniques for Nonviable Tissue Quantification in Late Gadolinium Enhancement Cardiac Magnetic Resonance Images
}

\author{
M. Chiara Carminati, PhD, ${ }^{*}$ Cinzia Boniotti, MS, $\dagger$ Laura Fusini, MS, ${ }^{*}$ \\ Daniele Andreini, PhD, * Gianluca Pontone, PhD, * Mauro Pepi, MD,* \\ and Enrico G. Caiani, PhD†
}

\begin{abstract}
Purpose: The aim of this study was to compare the performance of quantitative methods, either semiautomated or automated, for left ventricular (LV) nonviable tissue analysis from cardiac magnetic resonance late gadolinium enhancement (CMR-LGE) images.

Materials and Methods: The investigated segmentation techniques were: (i) n-standard deviations thresholding; (ii) full width at half maximum thresholding; (iii) Gaussian mixture model classification; and (iv) fuzzy c-means clustering. These algorithms were applied either in each short axis slice (single-slice approach) or globally considering the entire short-axis stack covering the LV (global approach). CMR-LGE images from 20 patients with ischemic cardiomyopathy were retrospectively selected, and results from each technique were assessed against manual tracing.
\end{abstract}

Results: All methods provided comparable performance in terms of accuracy in scar detection, computation of local transmurality, and high correlation in scar mass compared with the manual technique. In general, no significant difference between single-slice and global approach was noted. The reproducibility of manual and investigated techniques was confirmed in all cases with slightly lower results for the nSD approach.

Conclusions: Automated techniques resulted in accurate and reproducible evaluation of LV scars from CMR-LGE in ischemic patients with performance similar to the manual technique. Their application could minimize user interaction and computational time, even when compared with semiautomated approaches.

Key Words: cardiac imaging techniques, magnetic resonance imaging, myocardial infarction, image processing, computerassisted

(J Thorac Imaging 2016;00:000-000)

ate gadolinium enhancement cardiac magnetic reso- nance (CMR-LGE) is the standard imaging technique for the assessment of left ventricular (LV) myocardial viability, allowing the identification of scar tissue. ${ }^{1-4}$ With this

From the *Centro Cardiologico Monzino, IRCCS; and $†$ Department of Electronics, Information and Bioengineering, Politecnico di Milano, Milano, Italy.

The authors declare no conflicts of interest.

Correspondence to: M. Chiara Carminati, PhD, Centro Cardiologico Monzino, IRCCS, Via privata Carlo Parea 4, 20138 Milan, Italy (e-mail: mcarminati@ccfm.it).

Supplemental Digital Content is available for this article. Direct URL citations appear in the printed text and are provided in the HTML and PDF versions of this article on the journal's Website, www.thoracicimaging.com.

Copyright (C) 2016 Wolters Kluwer Health, Inc. All rights reserved.

DOI: $10.1097 /$ RTI.0000000000000206 procedure, a gadolinium-based contrast agent is injected, and after 10 to 20 minutes a single-frame sequence is acquired when the agent is washed-out by normal tissue, resulting in hyperenhanced (HE) intensity in nonviable myocardial tissue when compared with the darker normal myocardium.

The presence of scar tissue has an important prognostic and therapeutic value as a strong predictor of LV remodeling, cardiac dysfunction, and mortality. ${ }^{3,5-9}$ Its quantitative analysis is therefore of potential clinical interest and relies on the segmentation of the myocardial scar tissue in each image, where existing. To this end, several techniques for CMR-LGE image segmentation have been previously proposed. ${ }^{10-17}$ However, a recognized optimal method is still not defined, ${ }^{18}$ and in clinical practice the analysis is performed qualitatively using the 17-segment bull's eye model with a good agreement when compared with standard quantitative planimetry. ${ }^{19}$ Nevertheless, the extent of the HE scar tissue has been shown to be able to provide supplementary information beyond conventional risk stratification, ${ }^{20}$ thus suggesting that quantitative approaches should be used to measure scar extent and transmurality.

The most adopted computerized methods rely on threshold-based approaches, ${ }^{11,12,14,21-24}$ thus exploiting the enhanced intensity of the scar tissue compared with the surrounding normal myocardium. Besides manual tracing, recent recommendations ${ }^{18}$ proposed 2 semiautomatic threshold-based methods to segment HE regions, where the threshold is respectively defined as: (i) the intensity value $n$ standard deviations higher than the mean intensity of the normal myocardium (nSD); or (ii) the half value of the $\mathrm{HE}$ myocardium maximum intensity (full width at half maximum, FWHM). In both cases, user interaction is needed for each image to manually select suitable regions of interest (ROIs) used for calculating the threshold values.

The first aim of our work is to compare the performance of 4 different automated or semiautomated methods for nonviable tissue segmentation against reference standard manual tracing in CMR-LGE images acquired in ischemic patients. Furthermore, we aim to test the effect of deriving segmentation parameters by considering globally the entire short-axis (SA) stack (global approach), with benefit in increased analysis automation, compared with obtaining the same parameters singularly from each image (single-slice approach) as it is usually performed. In addition, we propose to quantify the presence and extent of nonviable tissue by automatically evaluating the local transmurality of myocardial scars in dedicated parametric representations. 


\section{MATERIALS AND METHODS}

\section{Imaging Data}

This study was approved by the institutional review board, and each patient gave her/his informed consent. We selected for the analysis cine and LGE CMR images of 20 patients ( 15 men, age $65 \pm 10 \mathrm{y}$ ) with previous myocardial infarction referred for the evaluation of LV function, which before this study had undergone the following acquisition protocol. Cine images were acquired during consecutive breath-holds using electrocardiogram-gated steady-state free precession sequence (1.5 T GE Healthcare, Discovery M450, 8-element phased-array coil, $512 \times 512$ matrix size, pixel spacing $0.74 \times 0.74 \mathrm{~mm}$, slice thickness $8 \mathrm{~mm}$, no overlap, no gap) in SA from the atrium to the apex. ECGtriggered LGE SA images were acquired during consecutive breath-holds using inversion recovery gradient echo scanning sequence $(256 \times 256$ matrix size, pixel spacing $1.4844 \times 1.4844 \mathrm{~mm}$, slice thickness $8 \mathrm{~mm}$, no overlap, no gap), 10 to 20 minutes after injection of an intravenous bolus of $0.1 \mathrm{mmol} / \mathrm{kg}$ gadolinium-gadobenate dimeglumine (Multihance-Bracco, Milan, Italy). Inversion time was individually adapted during acquisition to ensure intensity homogeneity between slices (usual range, 220 to $300 \mathrm{~ms}$ ). Images were acquired during systole, with delay after trigger in the range of 200 to $400 \mathrm{~ms}$.

\section{Segmentation Techniques}

All analyses and measurements were carried out using dedicated custom software developed in the Matlab environment (Mathworks Inc., Natick, MA). Four techniques were considered to segment nonviable myocardial tissue, previously proposed for HE scar quantification ${ }^{11,14,15,25}$ : (i) nSD thresholding (with $\mathrm{n}=3$ ); (ii) FWHM thresholding; (iii) automated Gaussian mixture model (GMM), including border zones (BZ) classification, the clinical importance of which has been previously reported ${ }^{26,27}$; and (iv) fuzzy cmeans clustering (FCM). Main characteristics of the investigated techniques are summarized in Table 1 (please refer to Hsu and colleagues ${ }^{11,14,15,25}$ for more detailed descriptions).

All these methods require the a priori knowledge of the myocardial position defined by its epicardial and endocardial borders, excluding papillary muscles, to define the regions of search for segmentation. Excluding this step, in the following text, we will refer to nSD and FWHM as "semiautomated" techniques, as user interaction is needed to define appropriate regions to define intensity thresholds, while the GMM and FCM will be referred as "automated" techniques, because no further user interaction is required.

\section{Postprocessing}

The following postprocessing operations were applied to refine results of all the segmentation techniques.
First, regions classified as HE were removed if their area was $<5 \%$ of the total myocardial area, likely representing noise. Furthermore, small dark areas surrounded by HE regions were identified as holes in the binary image resulting from segmentation and were included in the $\mathrm{HE}$ region, as potentially representing microvascular obstructions or intensity inhomogeneity of the HE signal. Finally, thin regions classified as $\mathrm{HE}$ connected to the epicardium or to the endocardium were removed because they could be potentially related to incorrect myocardial border delineation. The definition of these thin regions was made by computing the following threshold value:

$$
t r=\frac{\# \text { pixels connected to the myocardial border }}{\# \text { pixels constituting the scar area }} \times 100[\%]
$$

measuring for each segmented scar the percentage of pixels connected to one myocardial border with respect to the total scar size. Segmented scars connected to one myocardial border were removed if their $t r$ index reached the cutoff values:

$t r=100 \%$ for scars connected to the endocardium, meaning that all pixels belonging to the scar are connected to the endocardial border (scar thickness equal to 1 pixel); $t r \geq 50 \%$ for scars connected to the epicardium.

These values were empirically set, according to the rationale that classification errors are more prone to happen where segmented scars are connected to the epicardium, as scar position is expected to be subendocardial in the population of ischemic patients analyzed here.

This operation made it possible to remove pixels belonging to the blood pool or to the epicardial fat regions that can be erroneously classified as nonviable tissue if included in the myocardium during myocardial border delineation, as their intensity is comparable to scar tissue.

\section{Quantification of Scar Size and Transmurality}

After segmentation of the entire stack of SA images, the quantification of scar extent was estimated by computing the following indices:

- scar mass, i.e., the total volume of the nonviable tissue per slice, as sum of pixels in the HE area multiplied by the slice thickness, multiplied by the myocardial density $\left(1.05 \mathrm{~g} / \mathrm{cm}^{318}\right)$

- percent scar, i.e., the HE area for all SA images divided by the total amount of LV tissue (i.e. the area within the endocardial and epicardial contours for all images belonging to the SA stack)

- transmurality $(T)$, i.e., the percentage extent of nonviable tissue in the radial direction from the endocardium to the epicardium.

To compute $T$, each SA image was sampled with 2.5 degrees equally spaced line segments radially oriented starting from the center of the LV, estimated as the centroid

TABLE 1. Main Characteristics of the Investigated Segmentation Techniques

\begin{tabular}{lcccc}
\hline & Segmentation Approach & Parameters & Manual ROI & Border Zones \\
\hline nSD & Threshold-based & Threshold intensity $=3$ SD above dark myocardium & Yes & No \\
FWHM & Threshold-based & Threshold intensity $=50 \%$ of brightest myocardium & Yes & No \\
GMM & Cluster-based & $\begin{array}{c}\text { Histogram fitting with 3 Gaussian curves approximating } \\
\text { myocardial intensity }\end{array}$ & No & Yes \\
FCM & Cluster-based & $\begin{array}{c}\text { Probability cluster classification of myocardial intensities } \\
\text { based on fuzzy inference }\end{array}$ & No & No
\end{tabular}


of the region delimited by the epicardium. For each line segment, $T$ was then computed as the ratio between the radial length of the segmented scar and the myocardial local radial extent (Fig. 1). In clinical practice, the $T$ index is usually visually estimated referring to the 17-segment bull's eye model defined by the American Heart Association (AHA) ${ }^{28,29}$ In this model, the information is summarized for the basal, medial, and apical ventricular levels considering 3 representative SA slices, and transmurality is typically categorized into 3 classes $(T=0 \%, T<50 \%$, $T \geq 50 \%$ ), as shown in Figure 2 (left panel).

In this study, 2 automatically computed custom parametric representations are proposed. In the first one, named bull's eye low resolution (LRes), all the SA slices in the stack representing the LV, usually 7 to 10 images for each patient, are represented individually. In particular, each basal, medial, and apical slice is divided according to the coronary arterial perfusion territories into 6,6 , and 4 segments, respectively. Then, for each segment in which the scar covered $>50 \%$ of its circumferential extent, the maximum value of $T$ of all sampling profiles constituting the segment was computed and assigned to the segment on the basis of the classification $(T=0 \%, T<50 \%, T \geq 50 \%)$ used in clinical practice (Fig. 2, center panel). This representation differs from the AHA 17-segment model, as all segmented SA images are automatically analyzed and contribute to the analysis.

In the second parametric representation, a high-resolution (HRes) bull's eye model is proposed, potentially allowing for a more detailed description of the scar position and extent. The anatomic division into sectors is skipped, and each sampling profile is represented in the bull's eye model by visualizing the local value of $T$, according to a color-coded percentage scale from $0 \%$ to $100 \%$ (Fig. 2, right panel). The LRes and HRes bull's eye representations were automatically computed after segmentation for all investigated techniques and for the gold standard manual tracing.

\section{Analysis Protocol}

The reference technique for the algorithms performance comparison was constituted by the manual tracing of the HE scar regions in each SA image performed by a first experienced observer. As a first step, the myocardium was
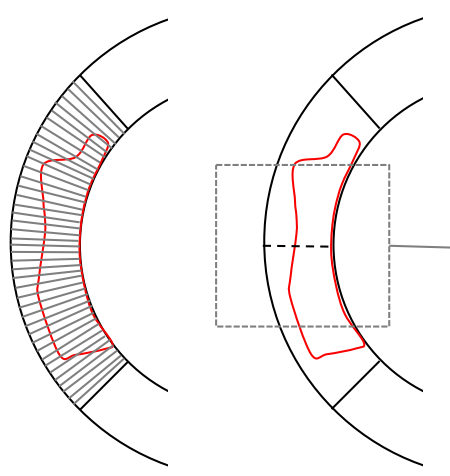

FIGURE 1. Schematic representation of transmurality computation: a myocardial sector with a scar region (in red) is sampled with radial profiles (left panel). For each profile (center panel), the transmural extent (right panel) is computed as the ratio between the scar width (green arrow) and the myocardium width (orange arrow) $\frac{\text { full color }}{\text { ouline }}$ defined by manually tracing both the endocardial and the epicardial contours. Cine images with corresponding slice position and cardiac phase were displayed as a reference alongside the LGE image to be contoured. Then, if present, scar regions were delimited by tracing their contours.

To avoid introducing variability in the evaluation of the different methods, the same endocardial and epicardial borders manually traced were used as region of search for all techniques.

Computerized analysis was performed as follows:

- for the automated methods (GMM and FCM), in the global approach the threshold values were computed by considering the pixels within the myocardial borders belonging to the entire SA stack, whereas in the single slice approach the threshold values were computed for each SA image separately;

- for the semiautomated methods (nSD and FWHM), in the global approach the SA stack was subdivided into 3 equal parts to define basal, medial, and apical portions. For each portion, the operator selected 1 representative slice on which the corresponding ROIs were manually traced, and thus used for computing the basal, medial, and apical threshold values. These values were applied for the analysis of slices belonging to the respective ventricular level. Conversely, in the single slice approach, the ROI selection and analysis were performed individually in each SA slice of the stack.

Algorithm performance was evaluated by comparing segmentation results with the manual technique, for both the single slice and the global approach. The correct identification of $\mathrm{HE}$ tissue presence was assessed by computing sensitivity, specificity, and accuracy for both the LRes and HRes representations by evaluating the presence of $\mathrm{HE}$ tissue in each sector and in each radial profile, respectively (true positive: HE for both the manual technique and the investigated method; false positive: HE for the investigated method only; true negative: normal myocardium for both the manual technique and the investigated method; false negative: $\mathrm{HE}$ for the manual technique but not by the investigated method).

The correct definition of $\mathrm{HE}$ areas was further evaluated by computing DICE similarity coefficient between the scar contours obtained by applying the implemented algorithms and those obtained by the manual technique (DICE $=2|X \cap Y| /(|X|+|Y|), \quad X=$ first contour, $\quad Y=$ second contour). Computational times required for the segmentation of the SA image stack for each method and approach were recorded.

Finally, repeatability analysis was assessed to evaluate the effect of observer subjectivity on HE measurements due to manual myocardial/scars contours tracings and ROI selections. A subset of 10 patients was randomly chosen and the entire analysis, including the manual tracing of myocardial borders, the scar contours and the ROI definition, was blindly repeated by the same observer, whereas the remaining 10 patients were analyzed by a second independent and experienced observer.

\section{Statistical Analysis}

For the accuracy study, Cohen $\kappa$ statistics was applied to measure the agreement of each method with the manual technique, for the single-slice and the global approaches. The McNemar test was applied to compare the approaches' sensitivity. 

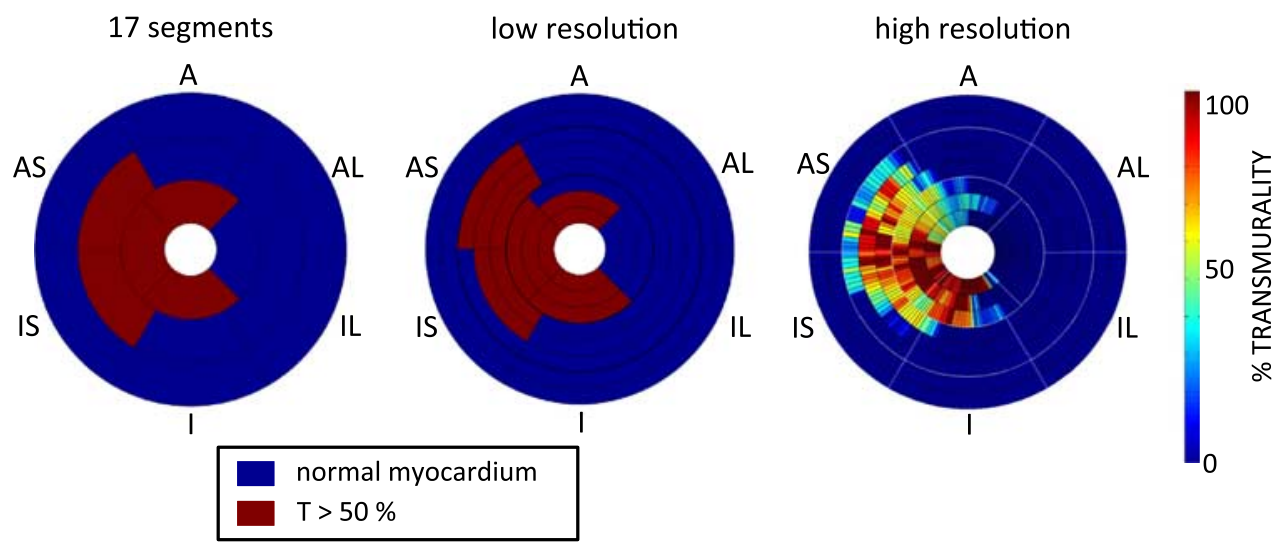

FIGURE 2. Bull's eye parametric representation of scar transmurality in SA CMR-LGE images. Left: conventional 17-segment model, in which results of scar segmentation in basal, mid, and apical regions are summarized by 1 ring each. Center: LRes model, in which results of scar segmentation in each SA image is reported as a separate ring. Right: HRes model, in which the transmurality value is computed along each $2.5^{\circ}$ equally spaced line segment. A indicates anterior; AL, antero-lateral; AS, antero-septal; I, inferior; IL, infero-lateral; IS, infero-septal. full color

Continuous variables (scar mass, percent scar, DICE similarity coefficient, and computational time) showed a non-normal distribution and were then expressed as median and 25 th, 75 th percentiles. The comparison between pairs of non-normal groups was made using Wilcoxon signed rank test, whereas Friedman statistics with Bonferroni correction was applied to test for differences in scar mass and percent scar measurements obtained with different methods, as appropriate.

The correct quantification of scar extent, measured as scar mass and percent scar, both for the single and global approach, was further verified by linear regression and Bland-Altman analyses for each method against the manual technique, wherein the significance of the bias was verified by applying paired Student $t$ test. To allow for homogeneity with the results of the other methods, BZs detected by the GMM approach were considered as normal myocardium in the analysis. For the correlation study, the $t$ test versus null was applied to test for the significance of the bias and the Fisher $z$ transformation was applied to test for differences in correlation coefficient values.

The derived HE measurements, i.e., scar mass and percent scar, were tested for interobserver and intraobserver variability by linear regression and Bland-Altman analysis, for both manual reference and investigated techniques. The coefficient of variation for each parameter was calculated as the SD of the signed difference between each pair of measurements divided by their mean value. For each method and each observer, the squared difference between the 2 observations was computed as an estimate of withinsubject variance for that method. ${ }^{30}$

For the variability study, Friedman test with Bonferroni correction was applied on the squared differences of the HE measurements to assess the reproducibility significance. ${ }^{30}$ Cohen $\kappa$ statistics were applied to measure the agreement between the repeated measures.

\section{RESULTS}

\section{Accuracy Analysis}

As expected, all patients showed HE tissues: 163 SA slices were overall analyzed and 77.3\% (126/163) had scars, wherein the percentage of slices with $\mathrm{HE}$ for each patient was in the range of $28.6 \%(2 / 7)$ to $100 \%(10 / 10)$.

Figure 3 shows an example of the proposed analysis in 1 representative patient: compared with the manual technique, all methods localized the $\mathrm{HE}$ tissue in the same position, with an extent that appeared to be slightly overestimated toward the infero-septal region for the nSD and GMM. Results obtained with single-slice and global approaches appeared very similar in all methods.

Accuracy analysis for both LRes (comparison on every sector) and HRes (comparison along each radial line) bull's eye representations resulted in high values of specificity, sensitivity and accuracy, with good agreement between each method and the manual approach (Table 2). In particular, in both single-slice and global approaches, specificity was high and very similar for the FWHM and GMM methods, whereas slightly degraded in nSD and FCM methods. Conversely, sensitivity was higher for nSD and FCM compared with FWHM and GMM. Significance of this finding was found for all cases except for the singleslice approach in the LRes bull's eye representation, in which FCM sensitivity result did not differ from those obtained from the other approaches. Total accuracy was slightly higher for the global approach than for the singleslice for the nSD, GMM, and FCM methods, with very similar results among the tested algorithms.

\section{Scar Mass}

Results for scar mass and percent scar values computed for each of the investigated methods showed no statistically significant difference between single-slice and global approach considering the same segmentation method, whereas for both single-slice and global approach, for both scar mass and percent scar, higher values were observed for the nSD and FCM approaches compared with those obtained with FWHM and GMM (Fig. 4). In all cases, no difference was found against the manual technique.

Table 3 shows the results of correlation and BlandAltman analyses against the manual technique for scar mass and percent scar indices, respectively. Correlation for scar mass index was between 0.64 and 0.73 , with no significant differences between single-slice and global 

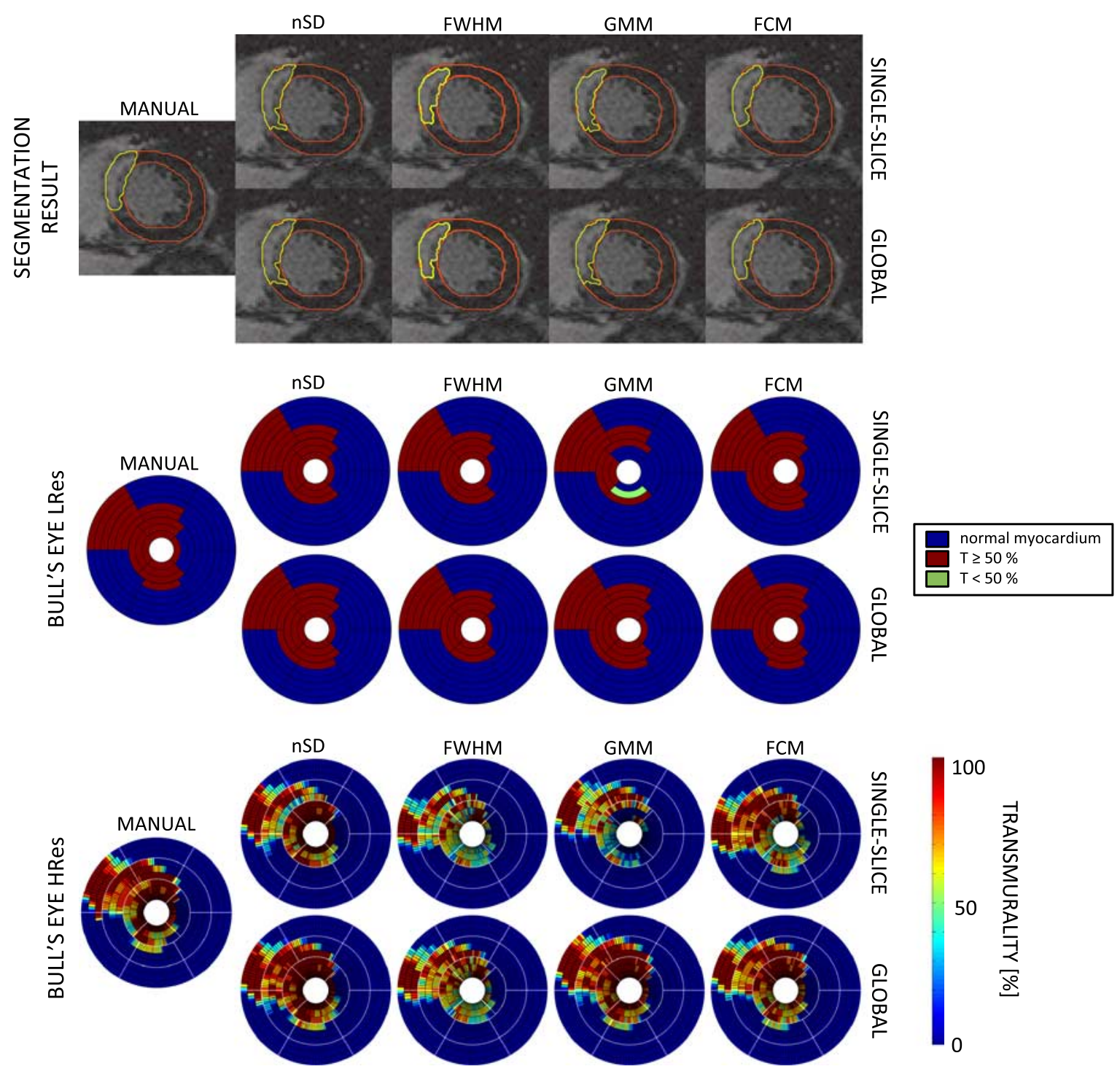

FIGURE 3. Example of the proposed analysis in 1 representative patient. Top: segmentation results in an SA slice. Mid: results of the SA stack analysis expressed as bull's eye LRes representation. Bottom: results of the SA stack analysis expressed as bull's eye Hres representation. $\frac{\text { full color }}{0 \mathrm{nline}}$

TABLE 2. Accuracy Analysis for HE Myocardial Tissue Detection on SA Images of LRes and HRes Bull's Eye Representations Versus Manual Technique, for Both the Single-slice and the Global Approach

\begin{tabular}{|c|c|c|c|c|c|c|c|c|c|}
\hline \multirow[b]{2}{*}{ Method } & \multirow[b]{2}{*}{ Bull's Eye } & \multicolumn{4}{|c|}{ Single-slice Approach } & \multicolumn{4}{|c|}{ Global Approach } \\
\hline & & Specificity & Sensitivity & Accuracy & к & Specificity & Sensitivity & Accuracy & к \\
\hline \multirow[t]{2}{*}{$\mathrm{n}-\mathrm{SD}$} & LRes & 87.34 & 92.56 & 88.96 & 75.59 & 89.63 & 92.92 & 90.66 & 79.16 \\
\hline & HRes & 84.65 & 92.86 & 87.6 & 74.2 & 87.06 & 93.11 & 89.23 & 77.4 \\
\hline \multirow[t]{2}{*}{ FWHM } & LRes & 94.32 & 77.34 & 89.06 & 73.7 & 95.47 & 71.7 & 88.05 & 70.72 \\
\hline & HRes & 93.59 & 77.6 & 87.86 & 72.95 & 94.23 & 73.18 & 86.68 & 69.95 \\
\hline \multirow[t]{2}{*}{ GMM } & LRes & 94.61 & 75.08 & 88.55 & 72.27 & 95.91 & 75.56 & 89.56 & 74.62 \\
\hline & HRes & 93.21 & 74.63 & 86.55 & 69.88 & 94.77 & 76.12 & 88.08 & 73.23 \\
\hline \multirow{2}{*}{ FCM } & LRes & 84.86 & 86.41 & 85.34 & 67.54 & 88.47 & 83.92 & 87.05 & 70.59 \\
\hline & HRes & 80.35 & 85.12 & 82.06 & 62.68 & 85.94 & 86.24 & 86.05 & 70.42 \\
\hline
\end{tabular}

All values are expressed as percentage. 


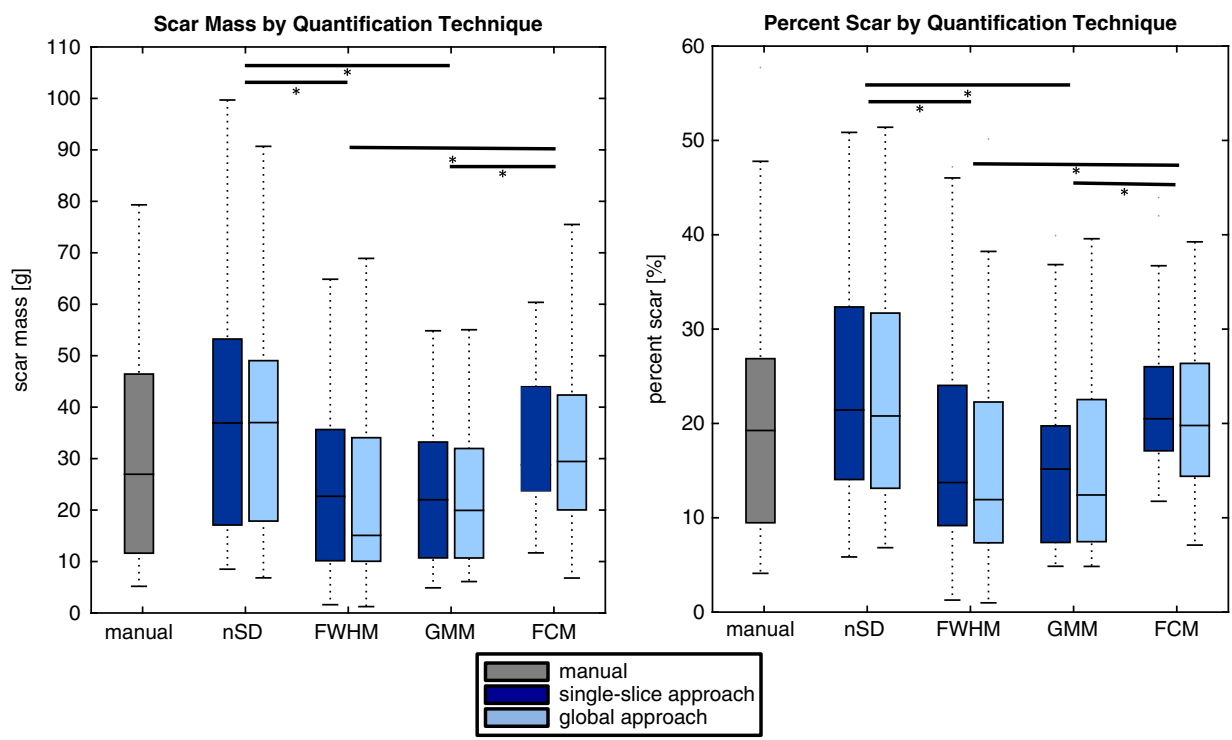

FIGURE 4. Cumulative results of scar mass (left) and percent scar (right) segmentation for each applied method, both for single-slice and global approach $\left({ }^{*} P<0.05\right.$ Friedman test). $\frac{\text { full color }}{2 n \operatorname{line}}$

approaches. When considering percent scar, correlation was higher in all cases, again with no significant differences between single-slice and global approach. For all methods, very limited biases were found (bias for scar mass $<1 \mathrm{~g}$, ranging from $1.5 \%$ to $6.6 \%$ for percent scar).

In all cases, DICE similarity coefficient (Table 4) resulted in high values $(>75 \%)$ with no significant differences between single-slice and global approaches.

\section{Computational Times}

Computational times required for the segmentation of the SA image was found to be significantly lower in all cases for automated techniques compared with those semiautomated, as well as in each method for the global approach compared with the single-slice segmentation (Supplemental Digital Content 1, http://links.lww.com/JTI/ A64). These results do not include the time required for the manual segmentation of the endocardial and epicardial contours, which was here considered as an a priori step for myocardial viability analysis.

\section{Variability Analysis}

For both intraobserver and interobserver analyses, very high and similar correlation coefficients for all tested approaches were found, with the highest correlation for the intraobserver analysis in the manual approach and for the interobserver analysis in the manual and nSD techniques (Supplemental Digital Content 2, http://links.lww.com/JTI/ A652). Bland-Altman analysis confirmed minimal interobserver and intraobserver biases and relatively narrow limits of agreement, with the lowest intraobserver bias in FCM and interobserver bias in GMM. Coefficient of variation values were remarkably lower for the percent scar compared with the scar mass in both intraobserver and interobserver analyses, suggesting that this global $\mathrm{HE}$ value is a more repeatable measure compared with the local scar mass. Cohen $\kappa$ analysis resulted in scattered values, showing, overall, a good agreement between repeated measurements with no noticeable differences among approaches.

Finally, the manual technique together with the automated methods (GMM and FCM) was found to be significantly more reproducible than the semiautomated

TABLE 3. Results of Correlation and Bland-Altman Analyses Computed for Scar Mass and Percent Scar Indices on SA Images Versus Manual Technique

\begin{tabular}{|c|c|c|c|c|}
\hline \multirow[b]{2}{*}{ Method } & \multicolumn{2}{|r|}{ Single-slice Approach } & \multicolumn{2}{|r|}{ Global Approach } \\
\hline & $\rho$ & Bias (g) $(95 \%$ Limits of Agreement) & $\rho$ & Bias (g) (95\% Limits of Agreement) \\
\hline \multicolumn{5}{|l|}{ Scar mass } \\
\hline n-SD & 0.73 & $-0.37^{*}(-1.5$ to 0.76$)$ & 0.73 & $-0.32 *(-1.4$ to 0.79$)$ \\
\hline FWHM & 0.65 & $0.2 *(-1$ to 1.4$)$ & 0.65 & $0.17^{*}(-1$ to 1.3$)$ \\
\hline GMM & 0.64 & $0.24 *(-0.97$ to 1.4$)$ & 0.69 & $0.19 *(-0.9$ to 1.3$)$ \\
\hline FCM & 0.72 & $0.058(-1$ to 1.2$)$ & 0.73 & $-0.067 *(-1.1$ to 0.95$)$ \\
\hline \multicolumn{5}{|c|}{ Percent scar } \\
\hline$n-S D$ & 0.83 & $-6.6^{*}(-23$ to 10$)$ & 0.86 & $-5.4^{*}(-19$ to 8.2$)$ \\
\hline FWHM & 0.69 & $3.5^{*}(-11$ to 18$)$ & 0.75 & $4.7^{*}(-6.7$ to 16$)$ \\
\hline GMM & 0.83 & $4.4^{*}(-7.8$ to 17$)$ & 0.87 & $4.4^{*}(-10$ to 19$)$ \\
\hline FCM & 0.76 & $-2.1(-17$ to 12$)$ & 0.78 & $-1.5(-14$ to 11$)$ \\
\hline
\end{tabular}

$\rho$ : Spearman correlation coefficient.

$* P<0.05, t$ test versus null for bias.

Copyright (C) 2016 Wolters Kluwer Health, Inc. Unauthorized reproduction of this article is prohibited.

This paper can be cited using the date of access and the unique DOI number which can be found in the footnotes. 
TABLE 4. DICE Similarity Coefficient, Expressed as Median and (25th, 75th) Percentiles for Each Segmentation Technique Computed Against Manual Reference

\section{DICE Similarity Coefficient (\%)}

\begin{tabular}{lcc} 
Method & Single-slice Approach & Global Approach \\
\hline nSD & $79.08(69.07,85.6)$ & $79.9(70.3,85.4)$ \\
FWHM & $76.9(68.4,83.2)$ & $76.5(62.6,84.7)$ \\
GMM & $75.2(63.6,83.7)$ & $79.4(65.9,85.6)$ \\
FCM & $78.6(68.6,85.6)$ & $78.5(68.7,87)$ \\
\hline
\end{tabular}

ones (nSD and FWHM), for intraobserver reliability, when computing scar mass with the global approach (Fig. 5, top panels). In general, for both intraobserver and interobserver analyses, the nSD was found to be the less reproducible technique (Fig. 5).

\section{DISCUSSION}

We have implemented and compared 4 different semiautomated or automated techniques for nonviable tissue quantification in LGE-CMR images, with dedicated

Intra-observer variability

Scar mass squared differences by quantification technique Percent scar squared differences by quantification technique
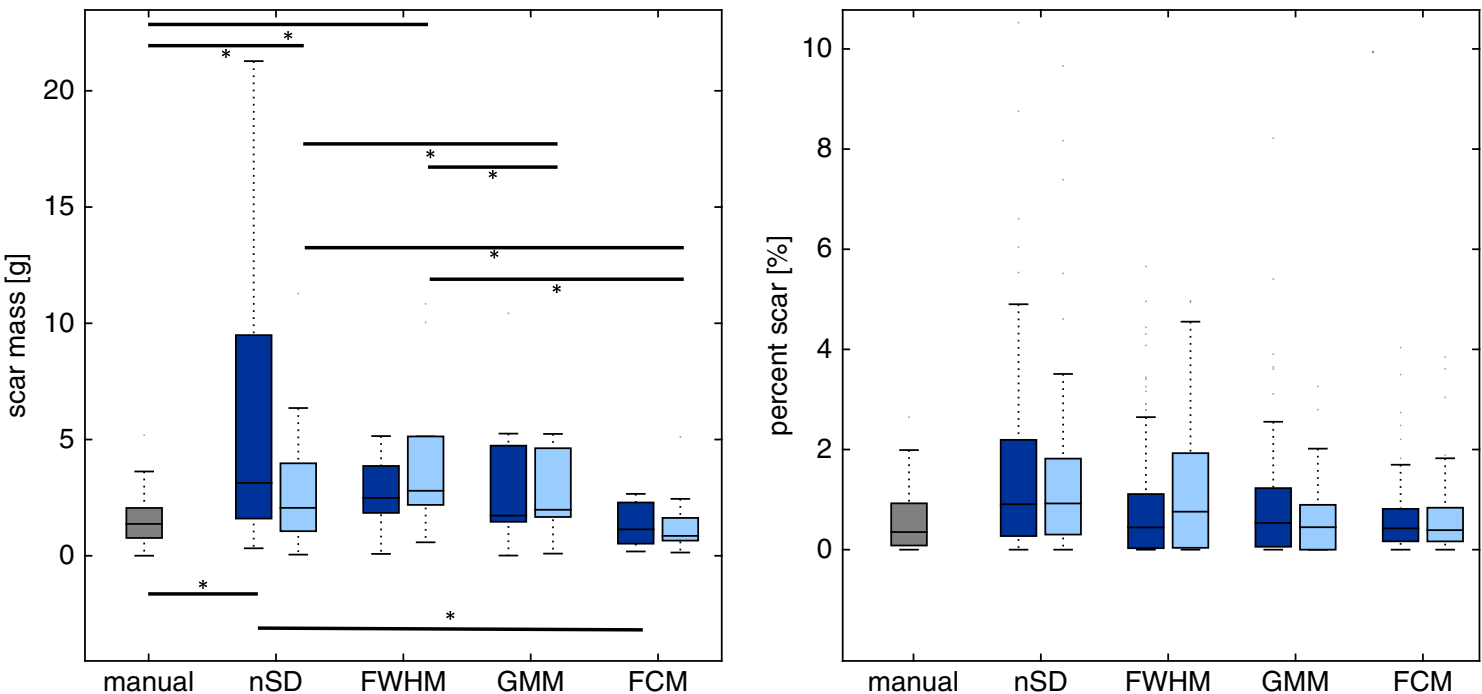

Inter-observer variability

Scar mass squared differences by quantification technique

Percent scar squared differences by quantification technique

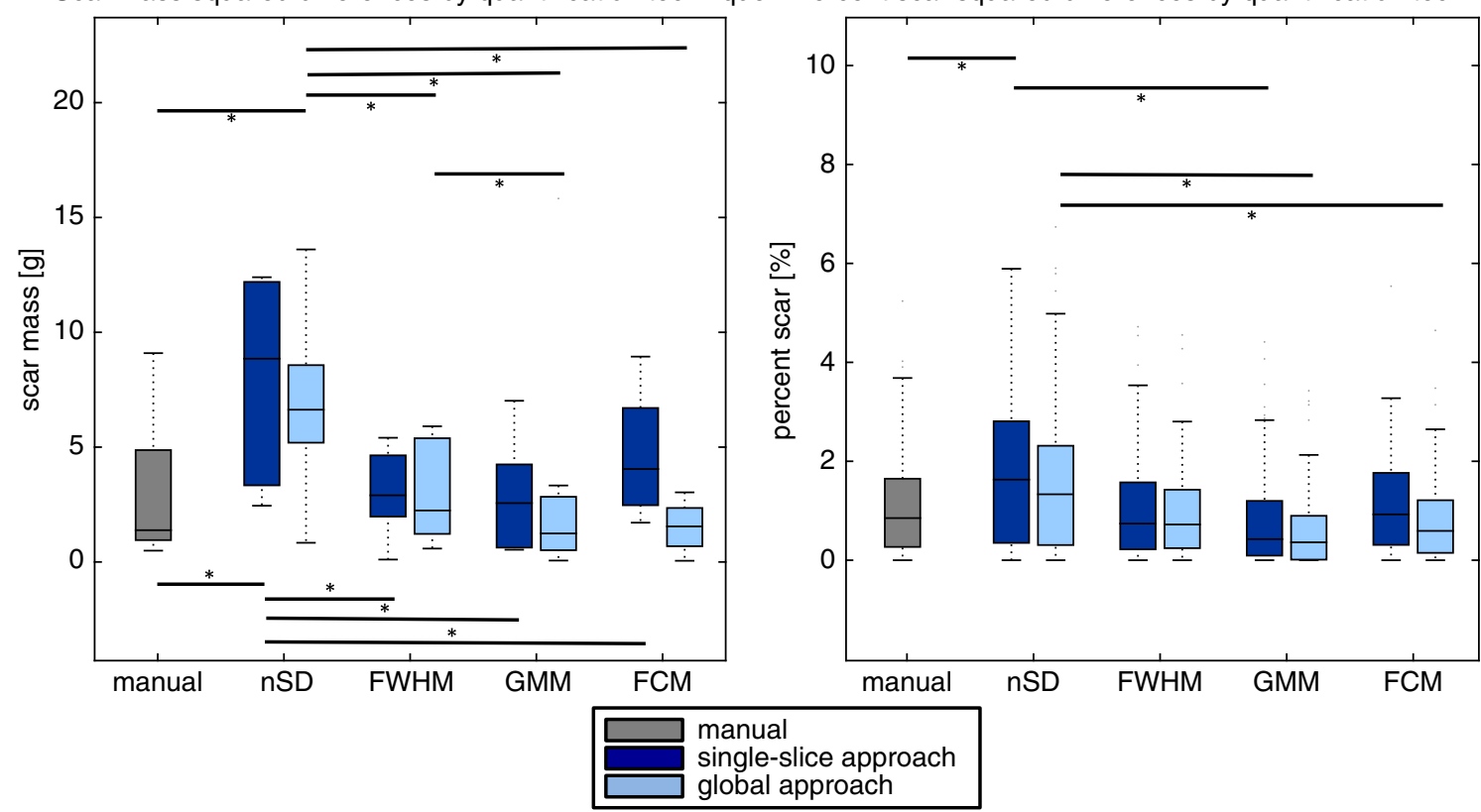

FIGURE 5. Intraobserver (top) and interobserver (bottom) reproducibility, expressed as the root squared differences of the repeated HE measurements, scar mass (left) and percent scar (right), for the manual techniques and each investigated method in both single-slice and global approaches $(* P<0.05$ Friedman test). full color

Copyright (C) 2016 Wolters Kluwer Health, Inc. All rights reserved. www.thoracicimaging.com | 7

Copyright (C) 2016 Wolters Kluwer Health, Inc. Unauthorized reproduction of this article is prohibited.

This paper can be cited using the date of access and the unique DOI number which can be found in the footnotes. 
postprocessing for segmentation refinement and quantitative evaluation of scar transmurality. Our main findings are that: (i) automated methods (GMM, FCM) resulted to be robust in detecting and quantifying nonviable tissue compared with the reference manual technique and the recommended semiautomated techniques (nSD, FWHM) and were also associated with higher intraobserver and interobserver reproducibility; (ii) a global approach in which segmentation was applied simultaneously to the myocardial regions in the whole SA stack or to subgroups of SA images led to reliable results with a significant decrease of user interaction and processing time.

On the basis of the published literature (Supplemental Digital Content 3, http://links.lww.com/JTI/A66, for a synthetic overview of published quantitative techniques for LGE image analysis), our analysis was focused on 2 popular semiautomated (nSD and FWHM) methods and on 2 automated (GMM and FCM) 2-dimensional methods.

The choice of nSD and FWHM was made as they are more frequently reported in both research papers and recent guidelines, ${ }^{3,18,21-23,25,31,32}$ wherein the FWHM was found to be the best method in terms of reproducibility and correlation with histopathology in animal models. ${ }^{31-32}$ However, both approaches need user interaction for manual delineation of suitable ROIs necessary for threshold computation with potential variability of the results due to subjective selection. For this reason, 2 automated approaches $\left(\mathrm{GMM}^{14,15}\right.$ and $\left.\mathrm{FCM}^{10,25}\right)$ were further investigated, resulting in objective and reproducible quantification of infarct size and extent in patients without the need of additional user interaction, thus suggesting that they should be preferred to the more common semiautomated techniques. Among the investigated techniques, GMM is the only method that allows for the segmentation of BZs, the clinical importance of which has been pointed out in several studies. ${ }^{27,33,34}$ However, further investigations are required to prove this assumption and quantify results on $\mathrm{BZ}$ segmentation, as this quantification was not available in the manual analysis, and thus its validation on GMM results was not possible.

Our results slightly differ from those presented by few other studies that comparatively investigated the ability of various methods to quantify viability from LGE images. ${ }^{25,32}$ Compared with these studies, we evaluated a cohort of patients with myocardial infarction, and we performed a more extensive validation based on infarct size measurements, DICE similarity coefficient, and accuracy analysis. Moreover, efforts were made to improve the local measurement of transmural extent of scar tissue, which was shown to significantly influence the response to resynchronization or revascularization therapies ${ }^{35,36}$ and whose large extent is associated with deleterious remodeling. ${ }^{37}$ Of note, no standard method has been defined to quantify transmural extent of scar myocardial tissue, and this value may vary significantly depending on the adopted technique. ${ }^{9}$

Our study showed the usefulness of a global approach to derive segmentation parameters to significantly decrease user interaction and computational time, as it does not require the subjective step of the a priori selection of slices showing HE tissue. Applicability of the global approach relies on homogeneity of the image intensities among slices, and, as previously reported, ${ }^{16}$ the usual pattern shows intensities that tend to become brighter when moving from the mitral valve plane to the apical slice. In our acquisitions, the manual adjustment of inversion times during acquisition ensured negligible intensity variations within LGE images $(0 \%$ to $8 \%$ for blood pool intensity and $0 \%$ to $6 \%$ for normal myocardium intensity), not visually appreciable.

Furthermore, recent developments in MR technology might envisage the use of $\mathrm{T} 1$ and $\mathrm{T} 2$ mapping sequence to further characterize the component of ischemic injury without the need of a contrast agent, as in LGE imaging. ${ }^{38,39}$ In this scenario, quantitative computerized approaches could play a significant role in the evaluation of the potential advantages of T1 and T2 sequences and their comparison with LGE.

The main limitation of our study is the lack of a true "gold standard" for the assessment of the HE measurements in CMR-LGE. This is, however, a difficult issue to address, as histologic validation in animal models considered as the gold standard in previous studies $3,11,14,21,31$ is actually an inadequate option due to differences in human and animal HE patterns, as already pointed out by Flett et $\mathrm{al}^{32}$ and Baron et al. $^{25}$ As a consequence, we decided to consider the manual technique, which proved to be repeatable, as the reference for accuracy and quantification analysis. Furthermore, scar segmentation was focused on LGE images of patients with ischemic cardiomyopathies, and thus dedicated postprocessing methods applied to all techniques were developed on the basis of the a priori knowledge of the typical morphologic characteristics of infarcted tissue (i.e. mostly connected tissue and in subendocardial position). However, nonischemic cardiomyopathies can also be studied through CMR-LGE, where the enhancement areas are characterized by unconnected zones of focal fibrosis. In these cases, postprocessing strategy should be optimized to handle the specific enhancement patterns.

In conclusion, we have proposed and compared quantitative methods for the analysis of nonviable tissue in CMR-LGE images for patients with ischemic cardiomyopathy. The global approach, jointly analyzing groups of SA slices or the entire SA image stack, led to faster and more reliable results compared with the classic single-slice approach and the manual gold standard. Among the investigated techniques, promising results were found for the automated methods, with a high level of accuracy and correlation with the manual gold standard, minimizing user interaction and the time required for the analysis.

\section{REFERENCES}

1. Judd RM, Lugo-Oliveri $\mathrm{CH}$, Arai M, et al. Physiological basis of myocardial contrast enhancement in fast magnetic resonance images of 2-day-old reperfused canine infarcts. Circulation. 1995;92:1902-1910.

2. Kim RJ, Chen E-L, Judd RM. Myocardial Gd-DTPA kinetics determine MRI contrast enhancement and reflect the extent and severity of myocardial injury after acute reperfused infarction. Circulation. 1996;94:3318-3326.

3. Kim RJ, Fieno DS, Parrish TB, et al. Relationship of MRI Delayed Contrast Enhancement to Irreversible Injury, Infarct Age, and Contractile Function. Circulation. 1999;100: 1992-2002

4. Mahrholdt H, Wagner A, Holly TA, et al. Reproducibility of chronic infarct size measurement by contrast-enhanced magnetic resonance imaging. Circulation. 2002;106:2322-2327.

5. Kim R, Wu E, Rafael A. The use of contrast-enhanced magnetic resonance imaging to identify reversible myocardial dysfunction. N Engl J Med. 2000;343:1445-1453. 
6. Kolipaka A, Chatzimavroudis G, White R, et al. Relationship between the extent of non-viable myocardium and regional left ventricular function in chronic ischemic heart disease. J Cardiovasc Magn Reson. 2005;7:573-579.

7. Roes SD, Kelle S, Kaandorp TM, et al. Comparison of myocardial infarct size assessed with contrast-enhanced magnetic resonance imaging and left ventricular function and volumes to predict mortality in patients with healed myocardial infarction. Am J Cardiol. 2007;100:930-936.

8. Kelle S, Roes SD, Klein C, et al. Prognostic value of myocardial infarct size and contractile reserve using magnetic resonance imaging. J Am Coll Cardiol. 2009;54:1770-1777.

9. Alexandre J, Saloux E, Dugué AE, et al. Scar extent evaluated by late gadolinium enhancement CMR: a powerful predictor of long term appropriate ICD therapy in patients with coronary artery disease. J Cardiovasc Magn Reson. 2013;15:12.

10. Positano V, Pingitore A, Giorgetti A, et al. A fast and effective method to assess myocardial necrosis by means of contrast magnetic resonance imaging. J Cardiovasc Magn Reson. 2005;7:487-494.

11. Hsu L-Y, Natanzon A, Kellman P, et al. Quantitative myocardial infarction on delayed enhancement MRI. Part I: animal validation of an automated feature analysis and combined thresholding infarct sizing algorithm. J Magn Reson Imaging. 2006;23:298-308.

12. Hennemuth A, Seeger A, Friman O, et al. A comprehensive approach to the analysis of contrast enhanced cardiac MR images. IEEE Trans Med Imaging. 2008;27:1592-1610.

13. Tao Q, Milles J, Zeppenfeld K, et al. Automated segmentation of myocardial scar in late enhancement MRI using combined intensity and spatial information. Magn Reson Med. 2010;64: 586-594.

14. Pop M, Ghugre NR, Ramanan V, et al. Quantification of fibrosis in infarcted swine hearts by ex vivo late gadoliniumenhancement and diffusion-weighted MRI methods. Phys Med Biol. 2013;58:5009-5028.

15. Hennemuth A, Friman O, Huellebrand M, et al. Mixture model-based segmentation of myocardial delayed enhancement MRI. In: MICCAI Workshop on Statistical Atlases and Computational Models of the Heart Imaging and Modelling Challenges (STACOM). 2013;7746:87-96.

16. Wei D, Sun Y, Ong S-H, et al. A comprehensive 3-d framework for automatic quantification of late gadolinium enhanced cardiac magnetic resonance images. IEEE Trans Biomed Eng. 2013;60:1499-1508.

17. Karim R, Chen Z, Obom S, et al. Infarct segmentation of the left ventricle using graph-cuts. In: MICCAI Workshoo on Statistical Atlases and Computational Models of the Heart Imaging and Modelling Challenges (STACOM). 2013;7746: 71-79.

18. Schulz-Menger J, Bluemke DA, Bremerich J, et al. Standardized image interpretation and post processing in cardiovascular magnetic resonance: Society for Cardiovascular Magnetic Resonance (SCMR) board of trustees task force on standardized post processing. J Cardiovasc Magn Reson. 2013;15:35.

19. Mewton N, Revel D, Bonnefoy E, et al. Comparison of visual scoring and quantitative planimetry methods for estimation of global infarct size on delayed enhanced cardiac MRI and validation with myocardial enzymes. Eur J Radiol. 2011;78:87-92.

20. Kwon DH, Halley CM, Carrigan TP, et al. Extent of left ventricular scar predicts outcomes in ischemic cardiomyopathy patients with significantly reduced systolic function. JACC Cardiovasc Imaging. 2009;2:34-44.

21. Fieno DS, Kim RJ, Chen EL, et al. Contrast-enhanced magnetic resonance imaging of myocardium at risk: distinction between reversible and irreversible injury throughout infarct healing. J Am Coll Cardiol. 2000;36:1985-1991.

22. Gerber BL, Garot J, Bluemke DA, et al. Accuracy of contrastenhanced magnetic resonance imaging in predicting improve- ment of regional myocardial function in patients after acute myocardial infarction. Circulation. 2002;106:1083-1089.

23. Setser RM, Bexell DG, O'Donnell TP, et al. Quantitative assessment of myocardial scar in delayed enhancement magnetic resonance imaging. J Magn Reson Imaging. 2003;18: 434-441.

24. Lund GK, Stork A, Saeed M, et al. Acute myocardial infarction: evaluation with first-pass enhancement and delayed enhancement MR imaging compared with 201Tl SPECT imaging 1. Radiology. 2004;232:49-57.

25. Baron N, Kachenoura N, Cluzel P, et al. Comparison of various methods for quantitative evaluation of myocardial infarct volume from magnetic resonance delayed enhancement data. Int J Cardiol. 2013;167:739-744.

26. Bello D, Fieno DS, Kim RJ, et al. Infarct morphology identifies patients with substrate for sustained ventricular tachycardia. J Am Coll Cardiol. 2005;45:1104-1108.

27. Yan AT, Shayne AJ, Brown K, et al. Characterization of the peri-infarct zone by contrast-enhanced cardiac magnetic resonance imaging is a powerful predictor of post-myocardial infarction mortality. Circulation. 2006;114:32-39.

28. Cerqueira MD, Weissman NJ, Dilsizian V, et al. Standardized myocardial segmentation and nomenclature for tomographic imaging of the heart a statement for healthcare professionals from the cardiac imaging committee of the Council on Clinical Cardiology of the American Heart Association. Circulation. 2002;105:539-542.

29. Ortiz-Pérez JT, Rodríguez J, Meyers SN, et al. Correspondence between the 17-segment model and coronary arterial anatomy using contrast-enhanced cardiac magnetic resonance imaging. JACC Cardiovasc Imaging. 2008;1:282-293.

30. Moon JC, Lorenz CH, Francis JM, et al. Breath-hold FLASH and FISP cardiovascular MR imaging: left ventricular volume differences and reproducibility 1. Radiology. 2002;223:789-797.

31. Amado LC, Gerber BL, Gupta SN, et al. Accurate and objective infarct sizing by contrast-enhanced magnetic resonance imaging in a canine myocardial infarction model. $J \mathrm{Am}$ Coll Cardiol. 2004;44:2383-2389.

32. Flett AS, Hasleton J, Cook C, et al. Evaluation of techniques for the quantification of myocardial scar of differing etiology using cardiac magnetic resonance. JACC Cardiovasc Imaging. 2011;4:150-156.

33. Schmidt A, Azevedo CF, Cheng A, et al. Infarct tissue heterogeneity by magnetic resonance imaging identifies enhanced cardiac arrhythmia susceptibility in patients with left ventricular dysfunction. Circulation. 2007;115:2006-2014.

34. Schuleri KH, Centola M, Evers KS, et al. Cardiovascular magnetic resonance characterization of peri-infarct zone remodeling following myocardial infarction. $J$ Cardiovasc Magn Reson. 2012;14:24.

35. White JA, Yee R, Yuan X, et al. Delayed enhancement magnetic resonance imaging predicts response to cardiac resynchronization therapy in patients with intraventricular dyssynchrony. J Am Coll Cardiol. 2006;48:1953-1960.

36. Ypenburg C, Roes SD, Bleeker GB, et al. Effect of total scar burden on contrast-enhanced magnetic resonance imaging on response to cardiac resynchronization therapy. $\mathrm{J} \mathrm{Am} \mathrm{Coll}$ Cardiol. 2007;99:657-660.

37. Hung CL, Verma $\mathrm{A}$, Uno $\mathrm{H}$, et al. Longitudinal and circumferential strain rate, left ventricular remodeling, and prognosis after myocardial infarction. J Am Coll Cardiol. 2010; 56:1812-1822.

38. Kellman P, Hansen MS. T1-mapping in the heart: accuracy and precision. J Cardiovasc Magn Reson. 2014;16:2.

39. Baeßler B, Schaarschmidt F, Stehning C, et al. Cardiac T2mapping using a fast gradient echo spin echo sequence-first in vitro and in vivo experience. J Cardiovasc Magn Reson. $2015 ; 17: 1-8$ 